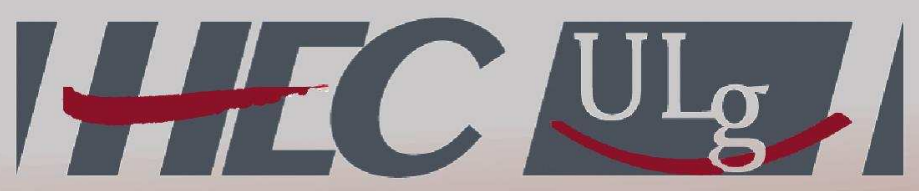

Ecole de Gestion de l'Université de Liège

CAHIER DE RECHERCHE / WORKING PAPER

A note on the use of the Modiffed Value-at-Risk

Laurent Cavenaille and Thomas Lejeune

February 10 / N²01002/01 


\title{
A note on the use of the Modified Value-at-Risk*
}

\author{
Laurent Cavenaile ${ }^{1}$ and Thomas Lejeune ${ }^{2}$
}

\begin{abstract}
While the Modified Value-at-Risk (or Cornish Fisher Value-at-Risk) has been quite extensively used by practitioners and academics since its introduction, we show that it can be consistently used only over a limited interval of confidence levels. Confidence levels below $95.84 \%$ should never be used for the Modified Value-at-Risk to be consistent with investors' preferences for kurtosis. In addition, the use of higher confidence levels is restricted by the value of the skewness. Failure to respect these restrictions on confidence levels results in mistakenly assessing risk and potentially overweighting assets which exhibit undesirable properties in terms of higher moments.

JEL Classification: G11, G32

Keywords: Value-at-Risk, Cornish-Fisher, Modified Value-at-Risk, Consistency
\end{abstract}

*We are grateful to Georges Hübner for helpful comments.

${ }^{1}$ KBL Chair in Fund Industry, HEC-Université de Liège, Belgium. Address: HEC-ULg, Rue Louvrex 14, Bldg N1, B-4000 Liège, Belgium. Tel. (+32)42327432. E-mail: Laurent.Cavenaile@ulg.ac.be

${ }^{2}$ HEC-Université de Liège, Belgium. Address: HEC-ULg, Rue Louvrex 14, Bldg N1, B4000 Liège, Belgium. Tel. (+32)42327429. Email: TLejeune@ulg.ac.be 
Since its introduction by Favre and Galeano (2002), the Modified Value-atRisk (MVaR) based on a Cornish-Fisher expansion (Cornish and Fisher (1938)) has been quite extensively used by practitioners and academic researchers as a measure of risk which accounts for asymmetry and fat tails in asset returns. It has been early mentioned in the literature that the traditional mean-variance analysis built on Markowitz's framework (Markowitz (1952)) did not adequately reflect the empirical risk-return trade-off for financial assets. In the late 1960's, a growing literature discussed investors' preferences for the third moment (skewness) of return distribution (see among others Arditti (1967), Jean (1971) and Kraus and Litzenberger (1976)). More recently, Scott and Horvath (1980) and Fang and Lai (1997) have extended the analysis to the fourth moment (kurtosis). This literature finds that investors require a higher risk premium for assets exhibiting lower skewness and higher kurtosis (ceteris paribus). Consequently, one should account for third and fourth moments of return distribution in addition to volatility when measuring the risk of an asset. In this respect, the MVaR appears as an easy-to-compute measure of risk that accounts for moments up to order four. It has become a popular risk measure for (among others) alternative investments which typically exhibit non-Gaussian returns. Beside the fact that it is relatively easy to calculate, the MVaR has the advantages of sharing the same understandable interpretation as any Value-at-Risk and of appearing as a generalization of the delta-normal Value-at-Risk to higher moments. However, it should be noticed that the MVaR has the desired property with respect to investors' preferences for higher moments only over a limited interval of confidence levels. In this paper, we emphasize the properties of the Cornish-Fisher quantile approximation in the MVaR definition and determine a range of (meaningful) confidence intervals for which the MVaR is consistent with investors' preferences regarding higher moments.

\section{The Modified Value-at-Risk}

Based on a Cornish-Fisher expansion estimation of the quantiles of non-Gaussian distribution, the MVaR is defined as:

$$
M V a R_{1-\alpha}=\mu+Z_{C F, \alpha} \sigma
$$

where $1-\alpha$ is the confidence level of the MVaR, $\mu$ the potential rate of drift of the asset value, $\sigma$ is the standard deviation of asset returns and $Z_{C F, \alpha}$ is the Cornish Fisher approximation of the $\alpha \%$ quantile of the distribution.

$$
Z_{C F, \alpha}=Z_{\alpha}+\frac{1}{6}\left(Z_{\alpha}^{2}-1\right) S+\frac{1}{24}\left(Z_{\alpha}^{3}-3 Z_{\alpha}\right) K-\frac{1}{36}\left(2 Z_{\alpha}^{3}-5 Z_{\alpha}\right) S^{2}
$$

where $Z_{\alpha}$ is the $\alpha \%$ quantile of a standard normal distribution, $\mathrm{S}$ is the standardized skewness and $\mathrm{K}$ is the excess kurtosis ${ }^{3}$.

\footnotetext{
${ }^{3}$ In case of an asset displaying Gaussian returns (with zero skewness and excess kurtosis), it can be easily shown that the MVaR simply boils down to the delta-normal Value-at-Risk.
} 
In the rest of this paper, we focus on the properties of $Z_{C F, \alpha}$ which is the only parameter affected by the potential non-normality of return distribution. Since a decrease in skewness and/or an increase in excess kurtosis should result in increasing risk (ceteris paribus), we expect $Z_{C F, \alpha}$ to be an increasing function with respect to skewness and a decreasing function with respect to kurtosis ${ }^{4}$. We now review the conditions under which $Z_{C F, \alpha}$ displays these characteristics. The structure of the MVaR and more particularly of $Z_{C F, \alpha}$ allows us to investigate skewness and kurtosis separately.

\section{The effect of excess kurtosis and skewness on the MVaR}

It is relatively straightforward to analyze the impact of an increase in kurtosis on the risk measured by the MVaR. Taking the first derivative of $Z_{C F, \alpha}$, we obtain the following restriction for the MVaR to be consistent with investors' preferences:

$$
\frac{\delta Z_{C F, \alpha}}{\delta K}=\frac{1}{24}\left(Z_{\alpha}^{3}-3 Z_{\alpha}\right)<0
$$

Focusing on the negative values of $Z_{\alpha}$, we get the following constraint for the MVaR to be consistent:

$$
Z_{\alpha}<-\sqrt{3} \simeq-1.732
$$

As a result, the MVaR is inconsistent for confidence levels below $95.84 \%$.

We can perform the same analysis for the skewness. The first derivative of $Z_{C F, \alpha}$ with respect to $\mathrm{S}$ gives the following constraint:

$$
\frac{\delta Z_{C F, \alpha}}{\delta S}=\frac{1}{6}\left(Z_{\alpha}^{2}-1\right)-\frac{1}{18}\left(2 Z_{\alpha}^{3}-5 Z_{\alpha}\right) S>0
$$

While $Z_{C F, \alpha}$ is linear function of excess kurtosis, it is a quadratic function of skewness. As a consequence, risk measured by the MVaR is decreasing in skewness only over limited intervals of skewness values. Moreover, for any confidence level of interest (e.g. confidence level which are frequently used in practice), we can determine a skewness value for which $Z_{C F, \alpha}$ (hence $M V a R_{1-\alpha}$ ) is minimum ${ }^{5}$ and below which the MVaR stops respecting the direction of the impact of skewness on risk imposed by investors' preferences.

\footnotetext{
${ }^{4}$ Since the MVaR is a negative value, any increase in risk corresponds to a decrease in the MVaR.

${ }^{5}-\frac{1}{36}\left(2 Z_{\alpha}^{3}-5 Z_{\alpha}\right)$ is positive for $\alpha$ between 0 and $5 \%$
} 


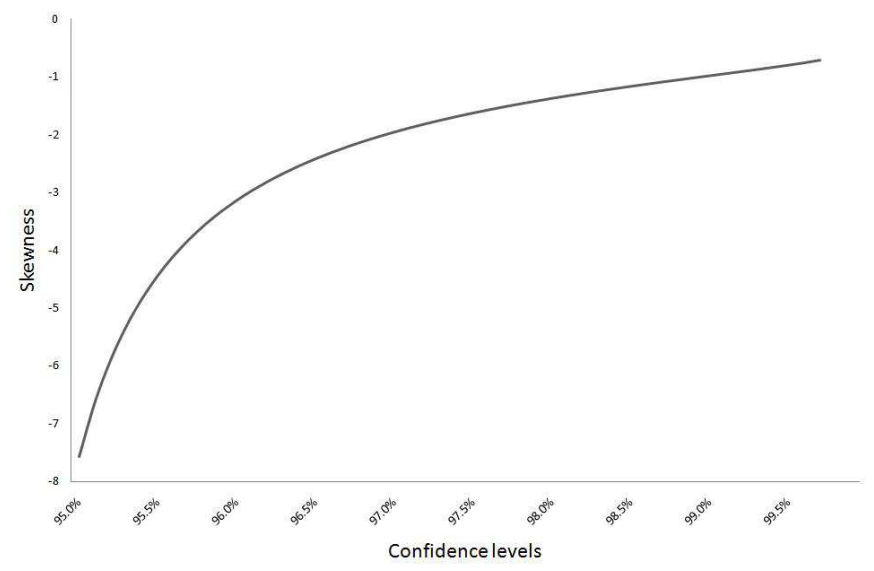

Figure 1: Minimum skewness for MVaR consistency as a function of confidence level

This minimum skewness is around -7.6 for the $95 \%$ confidence level whereas it is equal to -0.98 for the $99 \%$ confidence level. Consequently, the limit to the consistent use of the MVaR from the skewness point of view might appear to be restrictive since skewness values below -1 are not unrealistic in practice.

Table 1: Minimum skewness for MVaR consistency

\begin{tabular}{lrrrrr}
\hline Confidence level & $96.0 \%$ & $97.5 \%$ & $99.0 \%$ & $99.5 \%$ & $99.9 \%$ \\
\hline Minimum skewness & -3.13 & -1.62 & -0.98 & -0.79 & -0.59 \\
\hline
\end{tabular}

\section{Illustration}

We first illustrate the inconsistency of the MVaR for confidence levels below $95.84 \%$ focusing on the kurtosis. We determine the direction of the evolution of $Z_{C F, \alpha}$ (and hence of the MVaR) with respect to excess kurtosis for a fixed skewness ${ }^{6}$.

Without any loss of generality on the direction of the relation ${ }^{7}$, we set the skewness equal to zero. Figure 2 shows the relation between $Z_{C F, \alpha}$ and excess kurtosis for three commonly used confidence levels (95\%, 97.5\% and 99\%). As expected from our analysis, the direction of the evolution of $Z_{C F, \alpha}$ is not consistent with investors' preferences at the $95 \%$ confidence level.

\footnotetext{
${ }^{6}$ Our example satisfies the bounds within which the (excess) kurtosis can vary as a function of skewness (see for instance Teuscher and Guiard (1995) for a definition of this restriction).

${ }^{7}$ We have shown that the restriction on the confidence level due to kurtosis is not influenced by the level of skewness or kurtosis.
} 




Figure 2: Evolution of $Z_{C F, \alpha}$ as a function of excess kurtosis (skewness=0)

We perform the same analysis for the restriction coming from the skewness. However, in this case, the magnitude and occurrence of the inconsistency is a function of the skewness value. We compare the evolution of $Z_{C F, \alpha}$ as a function of skewness for an excess kurtosis of $5^{8}$. We report the results for 97.5 and $99 \%$ confidence levels $^{9}$ in Figure 3. We can see that the inconsistency occurs for values of skewness closer to zero as the confidence level increases hence decreasing the range of possible values of the skewness for the MVaR to remain consistent.

\section{Concluding remarks}

While the Modified Value-at-Risk (or Cornish Fisher Value-at-Risk) has been quite extensively used by practitioners and academics since its introduction, we show that it can be consistently used only over a limited interval of confidence levels. Confidence levels below $95.84 \%$ should never be used for the MVaR to be consistent with preferences for kurtosis. As a result, the broadly-used $95 \%$ confidence level leads to inconsistent results. In addition, the use of higher confidence level is restricted by the value of the skewness. In general, higher confidence levels impose more constraints on the admissible skewness levels for the MVaR to remain consistent. Failure to respect these restrictions on confi-

\footnotetext{
${ }^{8} \mathrm{An}$ excess kurtosis of 5 is a relatively frequent value especially for hedge funds' monthly returns while it allows for a relatively large range of possible skewness (see Teuscher and Guiard (1995)). Nevertheless, the results are similar for other values of excess kurtosis.

${ }^{9}$ We have already shown that the $95 \%$ confidence interval is always inconsistent regarding investors' preferences for kurtosis.
} 


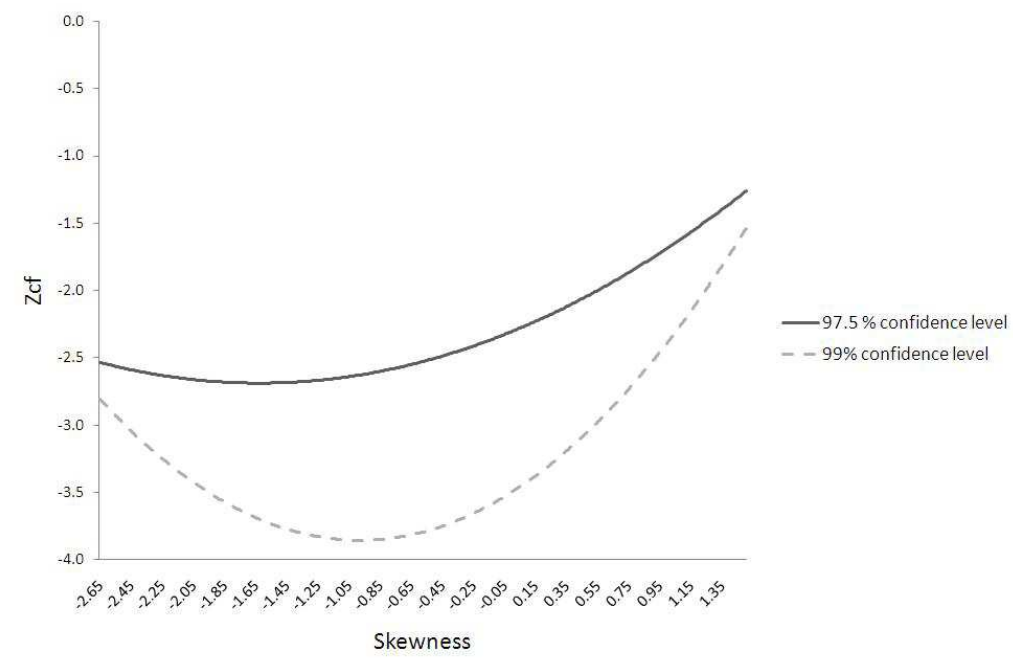

Figure 3: Evolution of $Z_{C F, \alpha}$ as a function of skewness (excess kurtosis=5)

dence levels results in mistakenly assessing risk and potentially in overweighting assets which exhibit undesirable properties in terms of higher moments.

\section{References}

Arditti, F. D. (1967). Risk and the required return on equity. The Journal of Finance, 22(1):19-36.

Cornish, E. A. and Fisher, R. (1938). Moments and cumulants in the specification of distribution. Review of the International Statistical Institute, 5(4):307320 .

Fang, H. and Lai, T.-Y. (1997). Co-kurtosis and capital asset pricing. The Financial Review, 32(2):293-307.

Favre, L. and Galeano, J.-A. (2002). Mean-modified value-at-risk optimization with hedge funds. Journal of Alternative Investments, 5(2):21-25.

Jean, W. H. (1971). The extension of portfolio analysis to three or more parameters. The Journal of Financial and Quantitative Analysis, 6(1):505-515.

Kraus, A. and Litzenberger, R. H. (1976). Skewness preference and the valuation of risk assets. The Journal of Finance, 31(4):1085-1100.

Markowitz, H. (1952). Portfolio selection. The Journal of Finance, 7(1):77-91. 
Scott, R. C. and Horvath, P. A. (1980). On the direction of preference for moments of higher order than the variance. The Journal of Finance, 35(4):915919.

Teuscher, F. and Guiard, V. (1995). Sharp inequalities between skewness and kurtosis for unimodal distributions. Statistics and Probability Letters, $22(3): 257-260$. 
Boulevard du Rectorat, 7 - Bât. B31 - 4000 Liège Rue Louvrex, 14 - Bât. N1 - 4000 Liège

\section{Belgium}

www.hec.ulg.ac.be 